N $84-29884$

NASA Technical Memorandum 85901

\title{
Flight Investigation of Various Control Inputs Intended for Parameter Estimation
}

Mary F. Shafer! 
NASA Technical Memorandum 85901

\section{Flight Investigation of Various Control Inputs Intended for Parameter Estimation}

Mary F. Shafer

Ames Research Center, Dryden Flight Research Facility, Edwards, California 93523

\section{NASก}

National Aeronautics and

Space Administration

Ames Research Center

Dryden Flight Research Facility

Edwards, California 93523 
FLIGHT INVESTIGATION OF VARIOUS CONTROL INPUTS INTENDED FOR PARAMETER ESTIMATION

\author{
Mary F. Shafer* \\ NASA Ames Research Center \\ Dryden Flight Research Facility \\ Edwards, California
}

\section{Abstract}

An experiment assessing the stability and control derivatives resulting from various control inputs was undertaken using the F- 8 digital fly-bywire aircraft. Improved control inputs have been proposed as a means of making stability and control derivative estimation more efficient, thus reducing the cost of flight testing and data analysis. The subject inputs were either generated by the pilot or preprogrammed in a remote ground computer and telemetered to the aircraft.

Nine preprogrammed inputs and three pilotgenerated inputs were assessed at subsonic and supersonic flight conditions, and both unaugmented and highly augmented flight control systems were used. Effects of input amplitude were also assessed. The inputs were divided into two general types - sinusoidal or with corners (a rapid and distinct change in slope).

The inputs with corners, performed in the unaugmented mode, produced the best sets of stability and control derivatives. The simplest of these inputs, the pilot-generated doublet, produced sets of derivatives as good as those produced by the more complex inputs.

Small inputs produced worse derivatives than larger inputs, the augmented mode resulted in worse derivatives than the unaugmented mode, and sinusoidal inputs produced worse derivatives than corner-containing inputs.

Use of the ground computer, with the control input telemetered to the aircraft, proved to be an efficient and useful technique for the generation of these inputs.

\section{Nomenclature}

All data are referenced to fuselage body axes according to right-handed sign conventions.

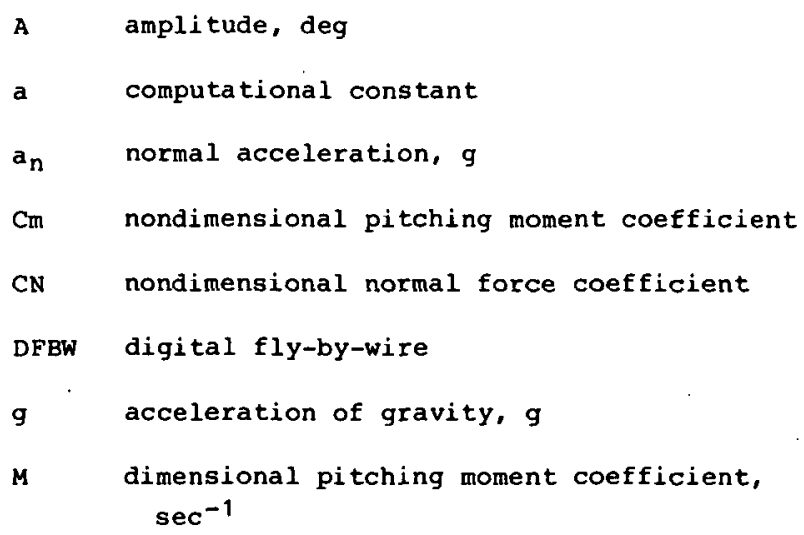
$\sec ^{-1}$

N

q pitch rate, $\mathrm{deg} / \mathrm{sec}$

RAV remotely augmented vehicle

T total time, sec

$t$ time, sec

SAS . stability augmentation system

$v$ velocity, ft/sec

a angle of attack, deg

Se elevator deflection, deg

$\theta$ pitch angle, deg

$\phi \quad$ bank angle, deg

$\omega \quad$ frequency, rad

Subscripts :

q rotary derivatives, per rad

$\alpha \quad$ static derivatives, per deg

Se control derivatives with respect to indicated quantity

$0 \quad$ initial

1 final

\section{Introduction}

The stability and control derivatives of an aircraft are used for simulators, for determining specification compliance, for validating design and wind tunnel studies, and in the design and refinement of flight control systems. This last use has become more important now that highly augmented aircraft are common because accurate values for the stability and control derivatives are necessary to design a satisfactory control system, unless the control system is unusually robust.

A first approximation of the stability and control derivatives can be estimated by computational techniques before the aircraft design is finalized. once the design has been set, better values for the stability and control derivatives can be determined by using a scale model of the aircraft in a wind tunnel. When the aircraft has been built, an accurate and complete set of derivatives for it can be determined from flight data. Just as the determination of the derivatives is a progressive process culminating in flight testing, so is the refinement of the flight control system. This refinement of the flight control system is based, 
in large part, on the increasingly accurate stability and control derivatives.

The determination of stability and control derivatives for an aircraft requires flight testing and extensive data analysis. Since both flight testing and data analysis are expensive and require skilled personnel and specialized facilities, any innovation that would reduce requirements wi thout reducing the quality of the stability and control derivatives would be welcomed. Since all parameter estimation techniques depend on a control input exciting the dynamics of the aircraft, attention has centered on the form of the control input, seeking the best, or optimal, input. This optimal input would produce the best response of the aircraft, with the greatest amount of information about the dynamics of the aircraft, for the determination of the complete set of stability and control derivatives. This, coupled wi th efficient data analysis techniques, would yield the best set of stability and control derivatives possible, and would make flight test more efficient. A variety of input forms have been proposed as optimal, based on theoretical considerations. $1,2,3,4,5$ However, many of these forms have not been assessed in flight test for a number of reasons. The most common reason is the inability of the pilot to precisely reproduce a desired, complex input. Because of this, only an approximation of the input can be assessed, and the relative merits of various inputs are obscured.

The most obvious way to solve the problem of desired input reproduction by the pilot is to bypass the pilot and make the input in another way. In the flight program described in Ref. 1, a system for applying the test inputs was installed in the aircraft. This system was composed of electrohydraulic actuators that were parallel to the normal aircraft control system, and a tape recorder for playing prerecorded test inputs to the actuators. Such a system is bulky and can only be used where extensive modification of the aircraft is possible. Another approach is to program the test inputs into the flight control system. This requires enough capacity in the flight control system and, for safety, requires extensive qualification of the modified flight control system. A third technique, the one used in the experiment described in this paper, is to use a flight control system based in a remote ground computer to generate the test inputs, which are telemetered to the onboard flight control system that has been modified to accept them.

These methods of bypassing the pilot require modification of the aircraft and are not always feasible. If pilot generation of the input signal is the only method available, then the best signal form, in terms of stability and control derivative estimation efficiency and in ease of replication, must be considered.

Another consideration in the assessment of these inputs is the influence of the flight control system. If, as is increasingly common, the augmentation cannot be turned of $f$ during these maneuvers, then the input must yield good stability and control derivatives for the augmented aircraft rather than for the bare airframe, as has previously been the case.
All of these elements discussed - interest in the effects of input form for highly augmented aircraft, the availability of a suitable aircraft, and the means of precisely reproducing the desired input - led to the flight program described in this paper. In this program, a variety of preprogrammed and pilot-generated inputs were assessed for the longitudinal axis. The preprogrammed inputs were examined in both augmented and unaugmented modes and were examined at subsonic and supersonic flight conditions. The pilot-generated inputs were examined in the unaugmented mode at the subsonic flight condition. This paper details the flight program, test procedure, and equipment used, and presents the results of the stability and control parameter estimation analysis.

\section{Flight Test Program}

This flight test program used the F- 8 digital fly-by-wire (DFBW) aircraft in conjunction with the remotely augmented vehicle (RAV) system to evaluate the proposed control inputs, which were evaluated in unaugmented and highly augmented control system modes. The test inputs, preprogrammed in the ground computer, were assessed by using the RAV flight control system, and the pilot-generated inputs were assessed by using the onboard flight control system.

\section{F-8 DFBW Aircraft and RAV System}

The F-8 DFBW aircraft is a standard F-8C aircraft that has been modified ${ }^{6}$ for research in digital fly-by-wire techniques, digital control system design and implementation, and handling and flying qualities studies. The modifications consisted of removing the entire mechanical control system between the stick and rudder pedals and the actuators. The mechanical control system was replaced by a digital fly-by-wire full-authority flight control system with a provision for accepting control surface commands from a ground-based computer while in a special RAV mode. 7,8

In the RAV mode, onboard control laws are bypassed and control laws that are programmed (in FORTRAN) in the ground computer are used instead. Necessary data are transmitted to the ground, and control surface commands are transmitted back to the aircraft, as shown in Fig. 1(a). The implementation of the unaugmented and augmented RAV modes are shown in Figs. $1(b)$ and $1(c)$. For comparison, the unaugmented onboard flight control system is shown in Fig. 1(d). The RAv mode is selected by the pilot. Failure detection and identification are accomplished in the ground and in the onboard computers; in the event of failure or malfunction the system is switched, automatically or manually, to the onboard control mode. Because the experimental RAV control laws are implemented in the ground computer, the integrity of the onboard flight control system is not disrupted. The flight qualification of the experimental control laws need not be as rigorous as that of the onboard control laws, thus providing a very cost-effective means of performing control-law research.

\section{RAV Control Modes}

Two RAV flight control modes, unaugmented and highly augmented, are used in this program. The 
highly augmented mode was developed for another experiment ${ }^{7}$ and used in. this program because of its availability and suitability. Both of these modes were modified to include a preprogrammed test input.

There are no feedback loops in the unaugmented mode, and the control surface command is the test input, as shown in Fig. 1(b). The highly augmented control mode uses the data transmitted from the aircraft to close the control loops, thus generating control surface commands, which are then transmitted back to the aircraft. As shown in Fig. 1(c), the control surface command is the test input plus the input generated by the augmentation. The RAV command is transmitted at a rate of 53.3 samples/sec, which is a function of the radio frequency of the uplink.

In this program the highly augmented control mode was a nonlinear pitch-rate controller, designed to maintain a damping level between 0.5 and 0.6 on the short period mode, while making the response speed as rapid as possible and minimizing the noise effects on the elevator.

\section{Onboard Control Modes}

Only one onboard flight control mode ${ }^{6}$ - the unaugmented mode - was used during evaluations of the pilot-generated inputs in this program. This unaugmented mode, known as DIRECT, has no feedback loops. Input shaping and gearing are performed in the onboard computers. The aircraft also has an augmented mode, which is a stability augmentation system (SAS) with feedback loops driven by the aircraft responses. The pilot uses an input panel in the cockpit to select the control system mode. The control modes of the pitch, roll, and yaw axes are selected separately. When longitudinal inputs are examined, the pitch axis is in DIRECT and the roll and yaw axes are in SAS.

\section{Preprogrammed Control Inputs}

There were nine preprogrammed control inputs available in this program. These inputs, shown in Fig. 2, are a doublet (Fig. 2(a)), a double doublet (Fig. 2(b)), a 3211 (Fig. 2(c)), a double 3211 (Fig. 2(d)), a subsonic multistep (Fig. 2(e)), a supersonic multistep (Fig. 2(f)), a short sine sweep of constant amplitude (Fig. $2(\mathrm{~g})$ ), a short sine sweep of increasing amplitude (Fig. 2(h)), a long sine sweep of constant amplitude (Fig. 2(i)), and a long sine sweep of increasing amplitude (Fig. $2(j)$ ). The inputs were coded with arbitrary amplitudes and scaling terms were included. The final values of the scaling terms were selected to give sufficiently large excitation of the airframe dynamics without exceeding the linearity assumptions of the equations of motion used in the estimation. These values were determined by using the F-8 DFBW fixed-base simulator.

The multiple inputs (double doublet and double 3211) were sized to have the same power as their respective single inputs; thus the amplitude of the multiple inputs was smaller than the single inputs. These multiple inputs were used at the subsonic flight condition to examine the effects of input amplitude and the linearity of the aircraft dynamics. In particular, the signal-to-noise ratio was lower for the smaller amplitude inputs. The
3211 input, named for the duration of each segment of the input, and documented in Ref. 1, has been used extensively in Europe. The multistep inputs were documented in an unpublished report by the authors of Ref. 3 and are based on the theory described therein.

The sine sweeps are defined by the equation:

$$
\delta e=A \sin \left(a w_{0} \ln (a-t)\right)
$$

where $a=\omega_{1} \mathrm{~T} /\left(\omega_{1}-\omega_{0}\right)$, and where the frequencies vary from $0.5 \mathrm{rad}$ to 4.0 rad in $9.6 \mathrm{sec}$ for the short sine sweep, and from $0.3 \mathrm{rad}$ to $10.0 \mathrm{rad}$ in 19.2 sec for the long sine sweep. For the constantamplitude sine sweeps the amplitude was unity, and for the increasing-amplitude sine sweeps it was $1+t / 2 T$. The constant and increasing amplitude sine sweeps were included to examine the effects of input power at the higher frequencies. Because of surface rate limits in the RAv system, the sine sweep is ramped between zero and the calculated magnitude for the first and last $1.5 \mathrm{sec}$. The ramp at the beginning of the long sine sweep is parabolic, and the ramps at the beginning of the short sine sweep and the end of both sine sweeps are linear.

As previously mentioned, the only constraints on exact replication of the proposed inputs are the actuator and control surface dynamics and signal limits that are part of the safety check routine of the RAV system. The most stringent safety limitation is on surface rate command, which limits the surface command rate to about 40 percent of the airplane surface rate. The actual surface rate is limited by actuator and surface dynamics.

\section{Pilot-Generated Inputs}

Three pilot-generated inputs, based on the preprogrammed inputs, were used. These inputs, shown in Fig. 3, are a doublet (Fig. 3(a)), a 3211 (Fig. 3(b)), and a sine sweep of constant amplitude (Fig. 3(c)). These inputs were chosen because it was feasible for the pilot to attempt to replicate them.

\section{Flight Test procedure}

The procedure for examining the various inputs is straightforward. Once the pilot reaches the target flight condition he selects the proper flight control system mode (DIRECT or RAV) and trims the aircraft. When pilot inputs are to be assessed, the pilot makes inputs, retrimming the aircraft between inputs as required. When preprogrammed inputs are to be assessed, the pilot makes a radio call when the aircraft is trimmed and he is ready. The engineer then initiates the ground computer input sequence. The appropriate RAV control system modes and input form and size are selected by this engineer, in accordance with the flight plan. If retrimming is required between inputs, the pilot does so before again indicating readiness. Multiple evaluations of preprogrammed and pilot-generated inputs are performed. Three evaluations of each input were planned. At any time, the pilot can disconnect the RAV system, reverting to an onboard control mode. The RAV system can also be disconnected by various safety checks. 
Data Collection and Analysis

The data that are measured and recorded for the F-8 DFBW aircraft include air data, Euler angles, angular rates, accelerations, control surface positions, RAV control surface commands, fuel quantity and angles of attack and sideslip. The air data parameters are used to calculate velocity, altitude, and Mach number. The fuel quantity is used to compute total weight, mass, center-ofgravity position, and moments of inertia.

The accelerometer signals are passed through noise-reduction filters before they are recorded. This filtering causes a time lag for these signals, relative to the other data, which can introduce inaccuracies into the stability and control derivatives. 9 Before analysis, these signals are timeshifted 10 to remove the lag introduced by the filters.

The computer program that is used to estimate the stability and control derivatives is named MMLE3. This program 11,12 is a modified maximum likelihood estimation program, capable of estimating the coefficients of linear differential equations and generating uncertainty levels for the coefficients. These uncertainty levels, or CramerRao bounds, provide statistical information about the quality of the coefficients, and are a guide to the relative accuracy of the coefficients. The MMLE3 program produces a time history (Figs. 4 and 5) of the flight and computed data for each maneuver. Computed values are produced using the input (control surface motion) with the mathematical model containing the estimated coefficients (the stability and control derivatives). The comparison of the measured values and the computed values provides information about the quality of the estimates. To have good estimates of the model coefficients, it is necessary to have a good match between the measured and computed time histories.

In addition to the time histories, summary plots are also produced (Fig. 6). The summary plots present the estimates and the uncertainty levels associated with each estimate. The match between the measured and computed time histories provides the first information about the quality of the estimates; however, more information is needed to assess the quality of the estimates, and this information is presented in the summary plots. The size of the uncertainty levels, indicated by the vertical bars on the plots, is an indication of the uncertainty in the estimate: The larger the bar, the more uncertainty in that particular estimate. Additional information about the accuracy of the estimates is provided by the scatter of the estimates.

The longitudinal.equations of motion used in this program are:

$$
\begin{aligned}
{\left[\begin{array}{l}
\dot{\alpha} \\
\dot{q} \\
\dot{\theta}
\end{array}\right]=} & {\left[\begin{array}{lll}
-\mathrm{N}_{\alpha} & 1 & (-\sin \theta)(\cos \phi) \mathrm{g} / \mathrm{V} \\
\mathrm{M}_{\alpha} & \mathrm{Mq} & 0 \\
0 & \cos \phi & 0
\end{array}\right]\left[\begin{array}{l}
\alpha \\
\mathrm{q} \\
\theta
\end{array}\right] } \\
& +\left[\begin{array}{ll}
-\mathrm{N}_{\delta} & { }^{-N_{0}} \\
\mathrm{M}_{\delta} & \mathrm{M}_{0} \\
0 & 0
\end{array}\right]\left[\begin{array}{l}
\delta \mathrm{e} \\
1
\end{array}\right] \\
\mathrm{a}_{\mathrm{n}} & =(\mathrm{V} / \mathrm{g}) \mathrm{N}_{\alpha} \alpha+(\mathrm{V} / \mathrm{g}) \mathrm{N}_{\delta e} \delta \mathrm{e}
\end{aligned}
$$

\section{Results}

Both pilot-generated and preprogrammed inputs have been examined at two flight conditions: Mach 0.6 (indicated) at 20,000 ft and Mach 1.2 (indicated) at $40,000 \mathrm{ft}$. A total of 71 maneuvers were performed. The details of these maneuvers are summarized in Table 1. At the subsonic flight condition, the inputs included pilot-generated inputs (assessed in DIRECT) and preprogrammed inputs (assessed in the unaugmented and highly augmented RAV control modes). All nine of the preprogrammed inputs were examined in the unaugmented RAV mode, and seven of the nine inputs were examined in the highly augmented RAV mode. The two inputs that were performed only in the unaugmented RAV mode were the double doublet and the double 3211; both were designed to assess the effect of input size. The assessment was not repeated in the augmented RAV mode because a previous study 13 has shown that for this aircraft, the stability and control derivatives determined from the unaugmented mode are better than those determined from the augmented mode.

At the supersonic flight condition, five of the nine preprogrammed inputs were examined. The inputs that were omitted were the two double inputs and the two long sine sweeps. Since time on the supersonic flight condition was at a premium, the long sine sweeps were omitted, partly because the results at the subsonic flight condition had indicated that these inputs were not suitable. The double inputs were not intended for assessment at this flight condition.

These maneuvers were analyzed using the MMLE program, and typical results are shown in Figs. 4 and 5. Figs. $4(a)$ to $4(c)$ show typical results for pilot inputs, Figs. 5(a) to 5(i) show typical results for the doublet, 3211 , multistep, and short and long sine sweep preprogrammed inputs performed in the unaugmented RAV mode, and Fig. $5(j)$ shows a typical result for the doublet performed in the augmented RAV mode. Examination of the pilot inputs in Fig. 4 shows that the pilot's doublet (Fig. 4(a)) and 3211 inputs (Fig. 4 (b)) do not match the ideal input in Fig. 3, since his inputs do not have the square corners that the ideal input has. This is particularly evident for the 3211 input. The attempted sine sweep (Fig. $4(\mathrm{C})$ ) resembles a sawtooth more than a sine. The differences between the commanded preprogrammed inputs telemetered to the aircraft and the actual elevator position (shown in Fig. 5) result from actuator and surface dynamics.

Figure 6 shows the summary plots of the derivatives estimated from the various results. These plots show both the estimated value of the derivative (the symbol) and the uncertainty level (the vertical bar) for each maneuver analyzed. The uncertainty level or Cramer-Rao bound gives an analytical estimate of the accuracy of the derivative. That is, the smaller the bound, the better the estimate. The scatter of the estimates of the derivative is also an indicator of the goodness of the estimates, with smalle-r scatter indicating better estimates. Thus to evaluate the suitability of an input shape, it is necessary to examine both the scatter and the uncertainty bound. 
To summarize the results, it can be seen that $\mathrm{Cm}_{\alpha}$ (Fig. 6(a)) is not particularly sensitive to input form or control mode, although it is sensitive to Mach number. In the unaugmented mode, $\mathrm{CN}_{\alpha}(\mathrm{Fig} .6(\mathrm{~b})$ ) is relatively insensitive to input form, but is more sensitive to form in the augmented mode. In Fig. $6(\mathrm{c})$, it can be seen that Cmoe is more sensitive to input form, particularly in the augmented mode. The other control derivative, CN $\delta$ e (Fig. 6(d)), is quite sensitive subsonically to input form and fairly insensitive to control mode. Supersonically, this derivative is quite insensitive to input form. Finally, $\mathrm{Cm}_{\mathrm{q}}$ (Fig. $6(\mathrm{e})$ ) is, like Cmoe' moderately sensitive to input form in the unaugmented mode and more sensitive to form in the augmented mode.

The best estimates of the complete set of stability and control derivatives resulted from the pilot-generated inputs and the nonsinusoidal preprogrammed inputs. For these inputs, the derivatives show little scatter and small uncertainty bounds compared with the derivatives from the other inputs. The element common to these inputs is what can best be described as a corner, or a rapid and distinct change in slope. The presence of these corners in the input leads to good estimates of the control derivatives. This means the less important derivatives are better estimated because good estimates of the control derivatives means less uncertainty, and therefore, better estimates of the less easily identified derivatives. Since the results appear to be relatively insensitive to input form as long as corners are present, good estimates of the derivatives can be obtained with relatively simple inputs such as doublets, as long as care is taken to generate a corner in the input. In confirmation of this, the derivatives from the pilotgenerated doublet show the highest scatter of any of the inputs with corners. Examination of the maneuvers for this input showed that the outlying values came from maneuvers in which the pilot had not generated corners as abrupt as those of the other inputs.

Comparison of the derivatives for the single and double doubiets and $3211 \mathrm{~s}$ (for the subsonic unaugmented mode) shows that the smaller double inputs yield poorer results (more scatter and larger uncertainty bounds) than do the larger single inputs. As previously mentioned, this result was expected. Because of the lower signal-to-noise ratio for the smaller amplitude input, more maneuvers with the smaller input are necessary to provide the amount of information provided by the maneuvers with the larger inputs, as long as these are not so large that they violate the linearity assumptions of the model. Thus it is necessary to have more of the poorer data instead of a lesser amount of good data to get acceptable results.

Analysis of all of the sine sweep inputs produced very poor matches between the computed and actual time histories (Fig. 5), indicating that the estimated values of the derivatives were not very good. The sine inputs are not suitable for generating the complete set of stability and control derivatives because the smoothly varying control input degrades the determination of the control derivatives. The poor natch between the computed and actual time histories is reflected in the greater scatter and larger uncertainty levels, seen in Fig. 6, for the derivatives estimated from the sine sweeps compared to the derivatives estimated from the inputs with corners. The best of the sine sweeps was the increasing amplitude short sine sweep, and the long sine sweeps were the worst; none of the sine sweeps were very good. However, there are applications, such as control system checkout, for which sinusoidal inputs are desirable. The major stability and control derivatives can be estimated from those maneuvers, but the quality of these derivatives will not be as good as the derivatives estimated from the same number of maneuvers from inputs with corners.

Another minor reason for poorer derivatives from these sinusoidal inputs is a violation of one of the assumptions of the model that the flight condition does not vary throughout the maneuver. This was not the case for all of these maneuvers. Rather, the altitude and Mach number varied, although slowly, during the maneuvers because of the nature of the input. The other inputs allowed the aircraft to remain trimmed, and the model assumption was not violated. The analysis program can compensate for this variation but at the expense of increased computation time.

Examination of the stability and control derivatives also yields information about the effect of the control system mode. Maneuvers performed in the unaugmented RAV mode produced better estimates of the complete set of stability and control derivatives than did maneuvers performed in the highly augmented RAV mode. This was not, as previously mentioned, unexpected. When augmentation is present there is a dramatic effect on the control input because the closure of the feedback loops changes the shape of the input. This change in input shape can be seen by comparing Fig. 5(j), which shows a doublet performed in the augmented RAV mode, with Fig. 5(a), which shows a doublet performed in the unaugmented RAV mode. In particular, the corners are removed from the input. The new form of the input is also less independent of the other aircraft parameters, and the amount of information contained in the maneuver is reduced. Again, as was the case for the small-amplitude inputs, satisfactory derivatives can be estimated by using the augmented mode, but more maneuvers will have to be analyzed than were analyzed for the unaugmented mode.

For this aircraft, it is not necessary to go to great lengths to devise and implement a complicated input form to optimize the estimation of the complete set of stability and control derivatives. Pilot-generated doublets yield a satisfactory set of longitudinal stability and control derivatives as long as the pilot is careful to generate corners in the input and make the input sufficiently large. In the opinion of the pilots, the doublet can be generated more easily than the more complex 3211 and sine sweep forms. One of the project pilots, when asked to comment on these inputs and their utility, said that the simplest input, because it is easiest to perform, could be more consistently performed when more than one pilot was involved. 
The most obvious conclusion from this experiment is that for this aircraft a broad class of control inputs produces complete sets of satisfactory longitudinal stability and control derivatives with little scatter and small uncertainty levels. The element common to all of these acceptable inputs is the presence of corners, or rapid and distinct changes in slope. Results from the preprogrammed and pilot-generated inputs indicate that as long as corners are present, relatively simple inputs, such as pilot-generated doublets, are the most efficient for obtaining good estimates of the derivatives. In addition, a pilot can perform simpler inputs more consistently than he can perform more complex forms.

Sinusoidal inputs, which do not have corners, do not produce derivatives as good as those from the inputs with corners. This is because the smoothly varying control input degrades the determination of the control derivatives.

Better derivatives result from maneuvers performed in the unaugmented, rather that augmented, control mode. This is because the shape of the input is changed by the augmentation (removing the corners), and because the information contained in the maneuver is reduced by the increased dependence between the control input and dynamic response parameters.

Small inputs do not produce derivatives as good as those from larger inputs, as long as the larger inputs are not so large that they cause violation of the linearity assumptions of the model. This is due to the lower signal-to-noise ratio of the small inputs.

Although the best sets of derivatives result from inputs with corners, used in the unaugmented mode, the derivatives can be estimated using small inputs or inputs performed in augmented modes. However, in order to get equally good derivatives from these three latter inputs, it is necessary to perform more maneuvers. Thus using these inputs is less efficient than using the inputs with corners in the unaugmented mode. Sinusoidal inputs can be used to determine only the major derivatives.

When analyzing the utility of various inputs, it is necessary to examine the entire set of stability and control derivatives because the major derivatives can be quite insensitive to factors that affect the other derivatives.

The RAV system, because of its flexibility and power, provided a very useful tool for the assessment of various inputs. Use of the RAV system ensured repeatable, precisely defined inputs so that the evaluation of the inputs was not obscured by imprecise versions of the inputs. Additionally, the use of the RAV system reduced checkout time because flight qualification was not necessary. l'plaetschke, E., and Schulz, G., "Practical Input Signal Design," AGARD-LS-104, Nov. 1979, PP. 3-1 to $3-19$.

${ }^{2}$ Cannaday, Robert L., and suit, william T., "Effects of Control Inputs on the Estimation of Stability and Control parameters of a Light Airplane," NASA TP-1043, 1977.

${ }^{3}$ Gupta, Narendra $K_{.}$, and Hall, w. Earl, Jr., "Input Design for Identification of Aircraft Stability and Control Derivatives," NASA CR-2493, 1975.

${ }^{4}$ Mehra, Raman K., "Optimal Input signals for Parameter Estimation in Dynamic Systems - Survey and New Results," IEEE Transactions on Automatic Control, vol. AC-19, no. 6, Dec. 1944, pp. 753-768.

${ }^{5}$ Stepner, David E., and Mehra, Raman K., "Maximum Likelihood Identification and optimal Input Design for Identifying Aircraft Stability and Control Derivatives," NASA CR-2200, 1973.

${ }^{6}$ Szalai, Kenneth J., Felleman, Philip G., Gera, Joseph, and Glover, Richard D., "Design and Test Experience with a Triply Redundant Digital Fly-bywire Control System," AIAA Guidance and Control Conference Proceedings, Aug. 1976.

${ }^{7}$ Larson, Richard R., Smith, Rogers E., and Krambeer, Keith D., "Flight-Test Results Using Nonlinear Control with the F-8C Digital Fly-by-Wire Aircraft," AIAA Guidance and Control Conference Proceedings, Aug. 1983, pp. 97-110.

8 Hartmann, Gary, Stein, Gunter, and Powers, Bruce, "Flight Test Experience with an Adaptive Control System Using a Maximum Likelihood Parameter Estimation Technique," AIAA Guidance and Control Proceedings, Aug. 1979, pp. 47-53.

${ }^{9}$ Steers, Sandra Thornberry, and Iliff, Kenneth w. " "Effects of Time-Shifted Data on FlightDetermined Stability and Control Derivatives," NASA TN D-7830, 1975.

10 Maine, Richard E., "User's Manual for SYNC, a FORTRAN Program for Merging and Time-Synchronizing Data," NASA TM-81355, 1981 .

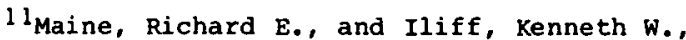
"User's Manual for MMLE3, a General FORTRAN Program for Maximum Likelihood Parameter Estimation," NASA TP-1563, 1980.

12 Iliff, Kenneth W., "Aircraft Identification Experience," AGARD-LS-104, Nov. 1979, Pp. 6-1 to 6-35.

${ }^{13}$ Shafer, Mary F., "Low-Order Equivalent Models of Highly Augmented Aircraft Determined from Flight Data," AIAA Journal of Guidance, Control, and Dynamics, vol. 5, no. 5, Sept.-Oct. 1982, pp. 504511 . 
Table 1 summary of flight activity.

\begin{tabular}{|c|c|c|c|}
\hline & \multicolumn{2}{|c|}{ Subsonic } & \multirow{2}{*}{$\begin{array}{l}\text { Supersonic } \\
\text { Unaugmented }\end{array}$} \\
\hline & Unaugmented & Augmented & \\
\hline \multicolumn{4}{|l|}{ Preprogrammed inputs } \\
\hline Doublet & 3 & 3 & 2 \\
\hline Double doublet & 3 & - & - \\
\hline 3211 & 3 & 3 & 3 \\
\hline Double 3211 & 3 & - & - \\
\hline Multistep & 3 & 2 & 2 \\
\hline Constant amplitude, short sine sweep & 3 & 3 & 3 \\
\hline Increasing amplitude, short sine sweep & 4 & 2 & 2 \\
\hline Constant amplitude, long sine sweep & 3 & 3 & - \\
\hline Increasing amplitude, long sine sweep & 3 & 2 & - \\
\hline \multicolumn{4}{|l|}{ Pilot-generated inputs: } \\
\hline Doublet & 9 & - & - \\
\hline 3211 & 2 & - & - \\
\hline Sine sweep & 2 & - & - \\
\hline
\end{tabular}

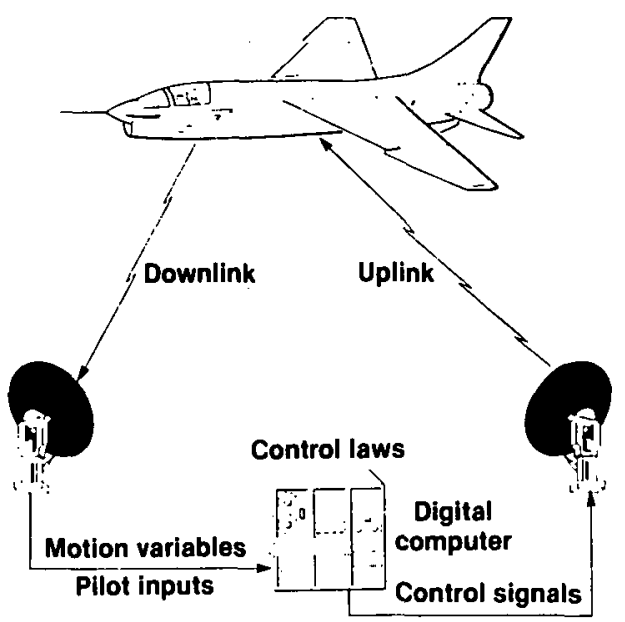

(a) Remotely augmented (RAV) test system.

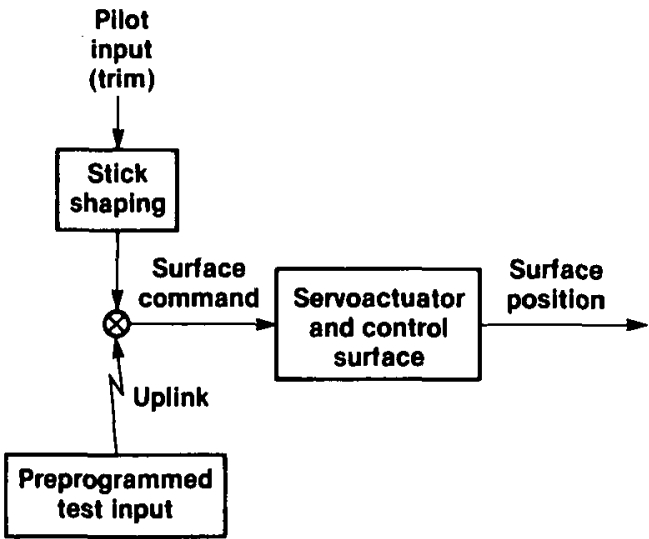

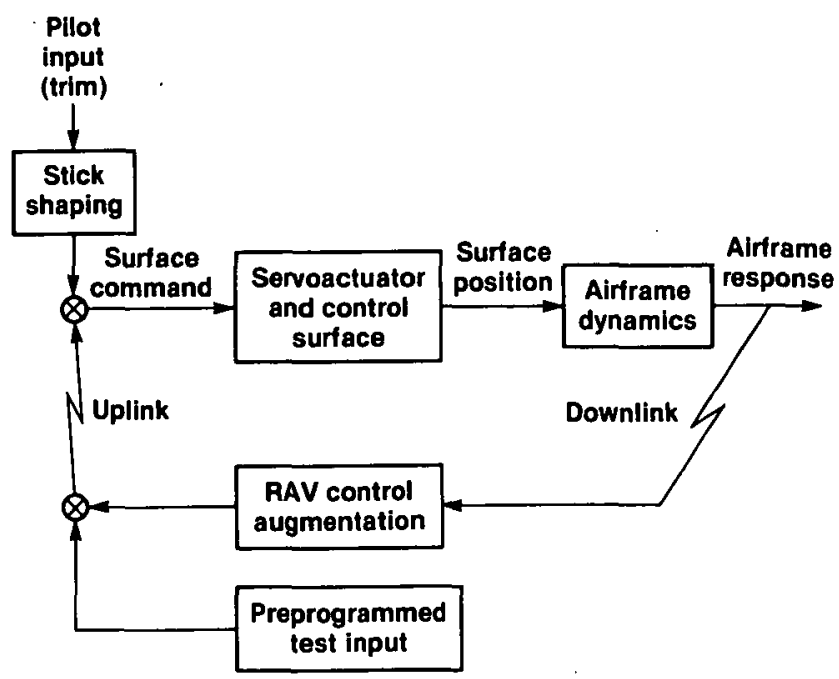

(c) Augmented RAV flight control system.

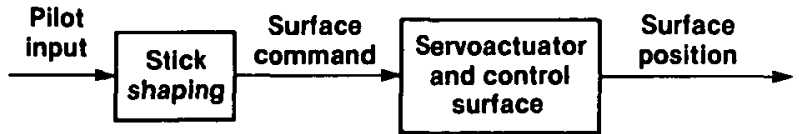

(d) Unaugmented onboard flight control system.

(b) Unaugmented RAV flight control system.

Fig. 1 Test system and flight control systems. 


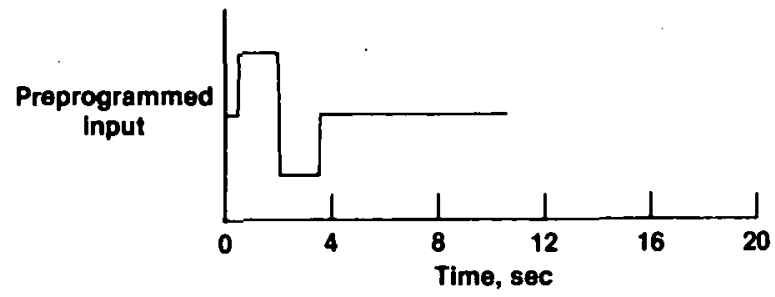

(a) Doublet.

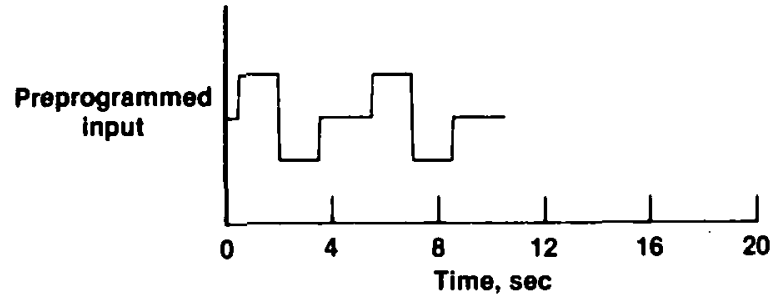

(b) Double doublet.

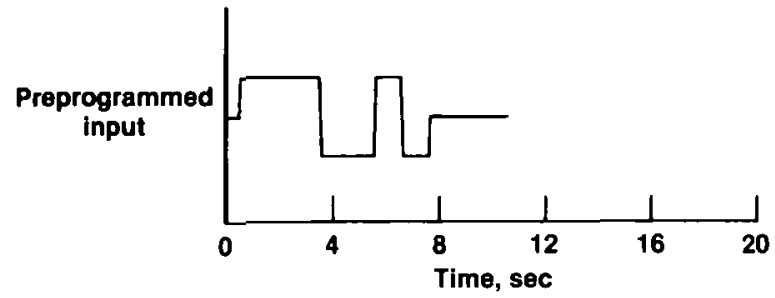

(c) 3211 .

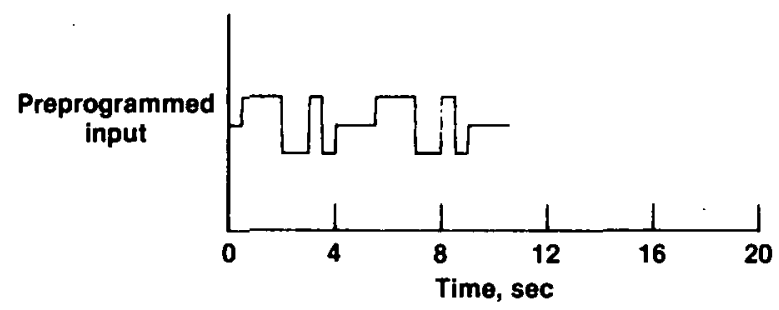

(d) Double 3211 .

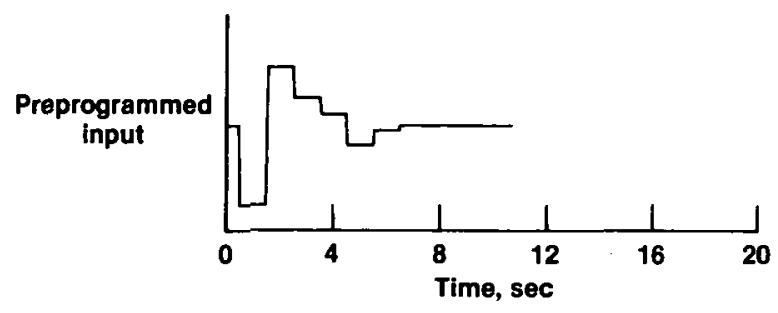

(e) Subsonic multistep.

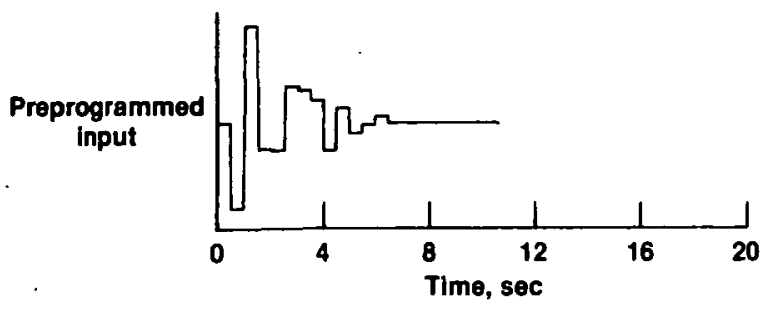

(f) Supersonic multistep.

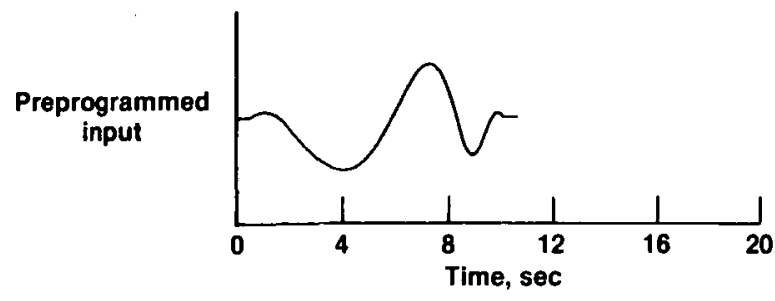

(g) Constant amplitude short sine sweep.

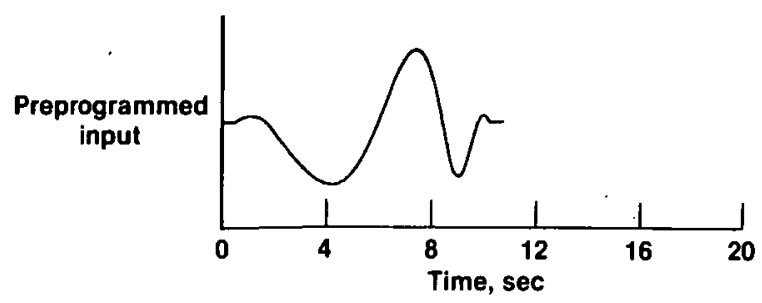

(h) Increasing amplitude short sine sweep.

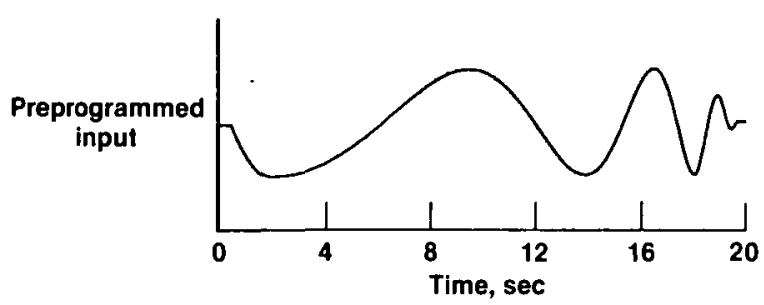

(i) Constant amplitude long sine sweep.

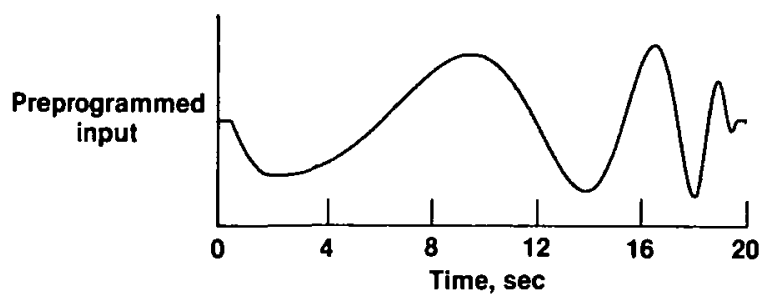

(j) Increasing amplitude long sine sweep.

Fig. 2 Preprogrammed test inputs. 


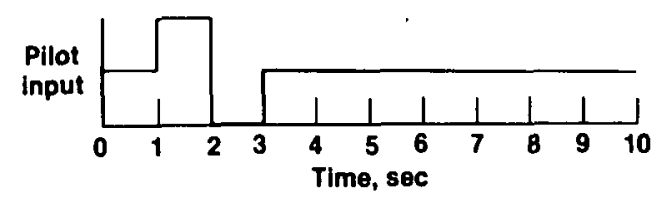

(a) Doublet.

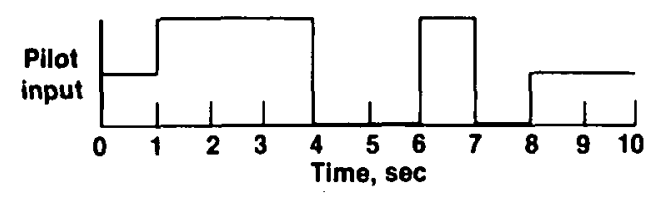

(b) 3211 .

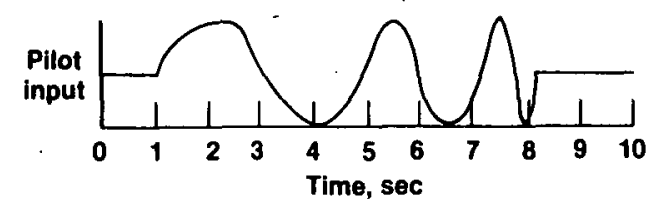

(c) Sine sweep.

Fig. 3 Ideal pilot inputs (f rom pilot's flight card).
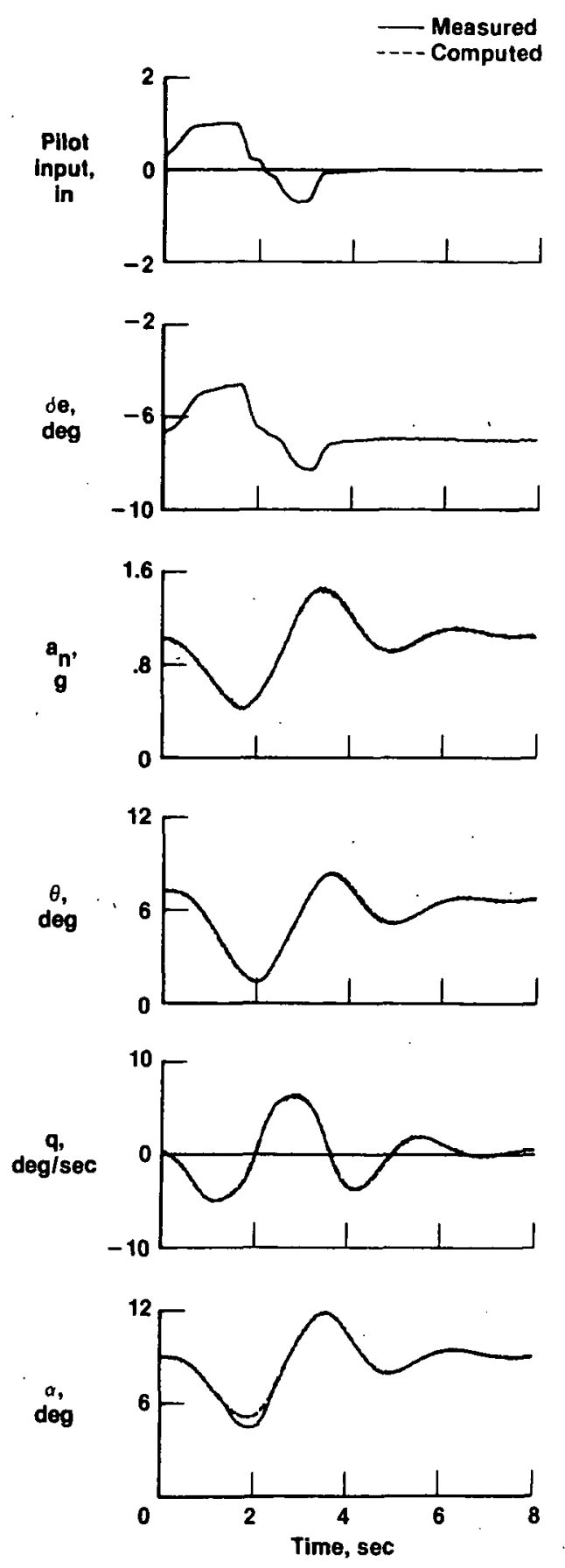

(a) Doublet input.

Fig. 4 Time histories for pilotgenerated inputs. 

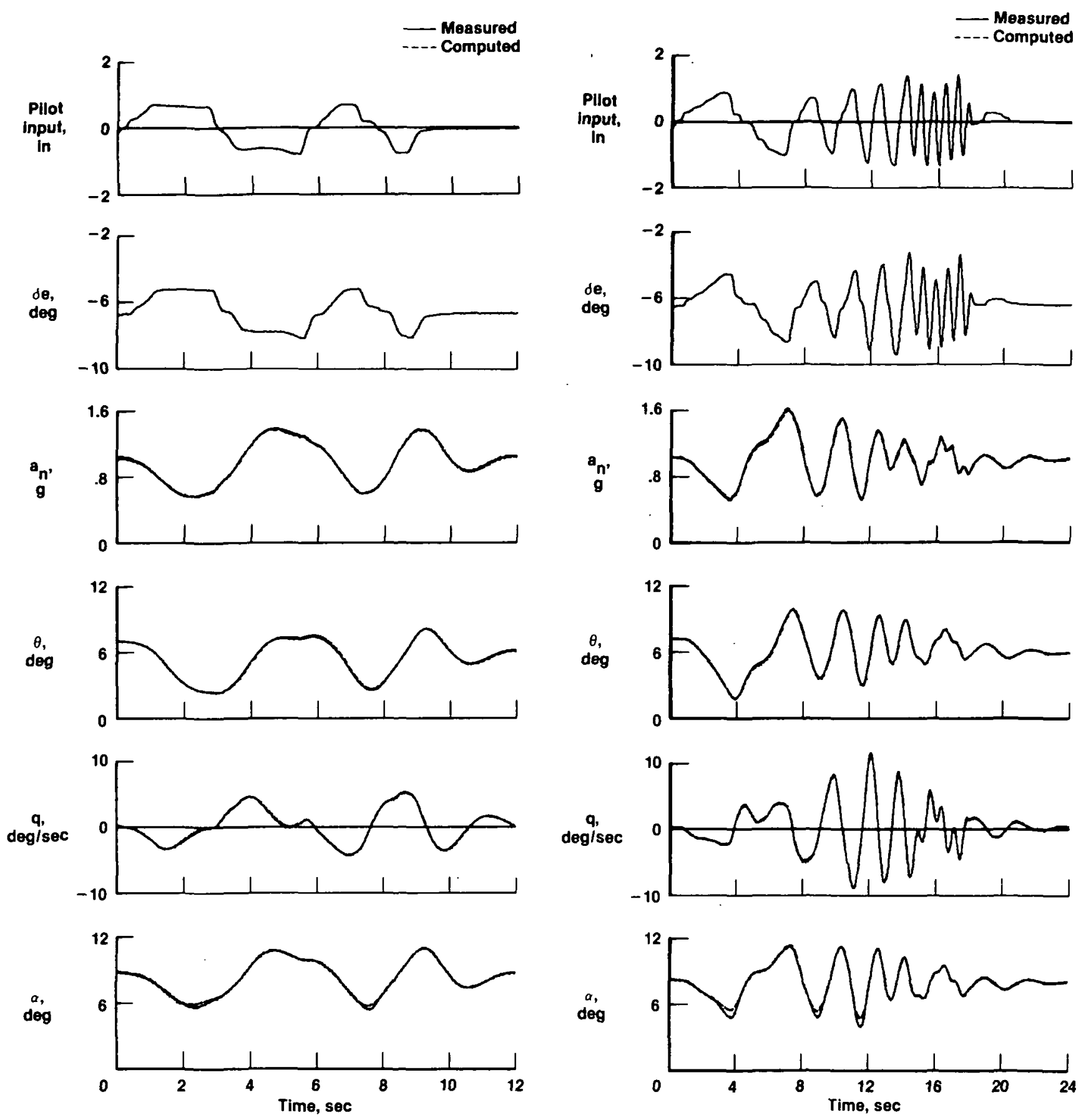

(b) 3211 input.

Fig. 4 Concluded.

(c) Sine sweep input. 

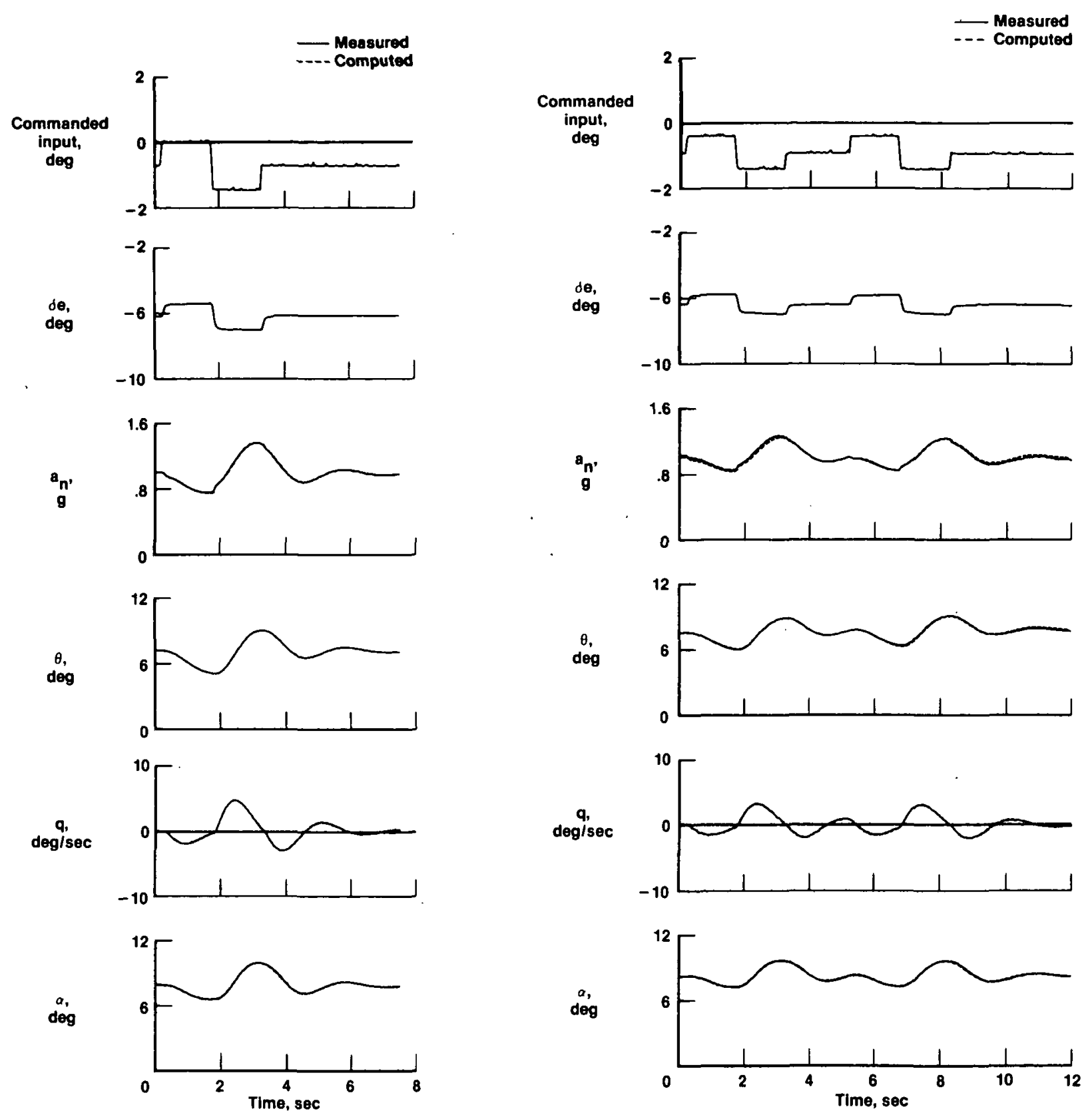

(a) Doublet input (unaugmented mode).

(b) Double doublet input (unaugmented mode).

Fig. 5 Time histories for preprogrammed inputs. 

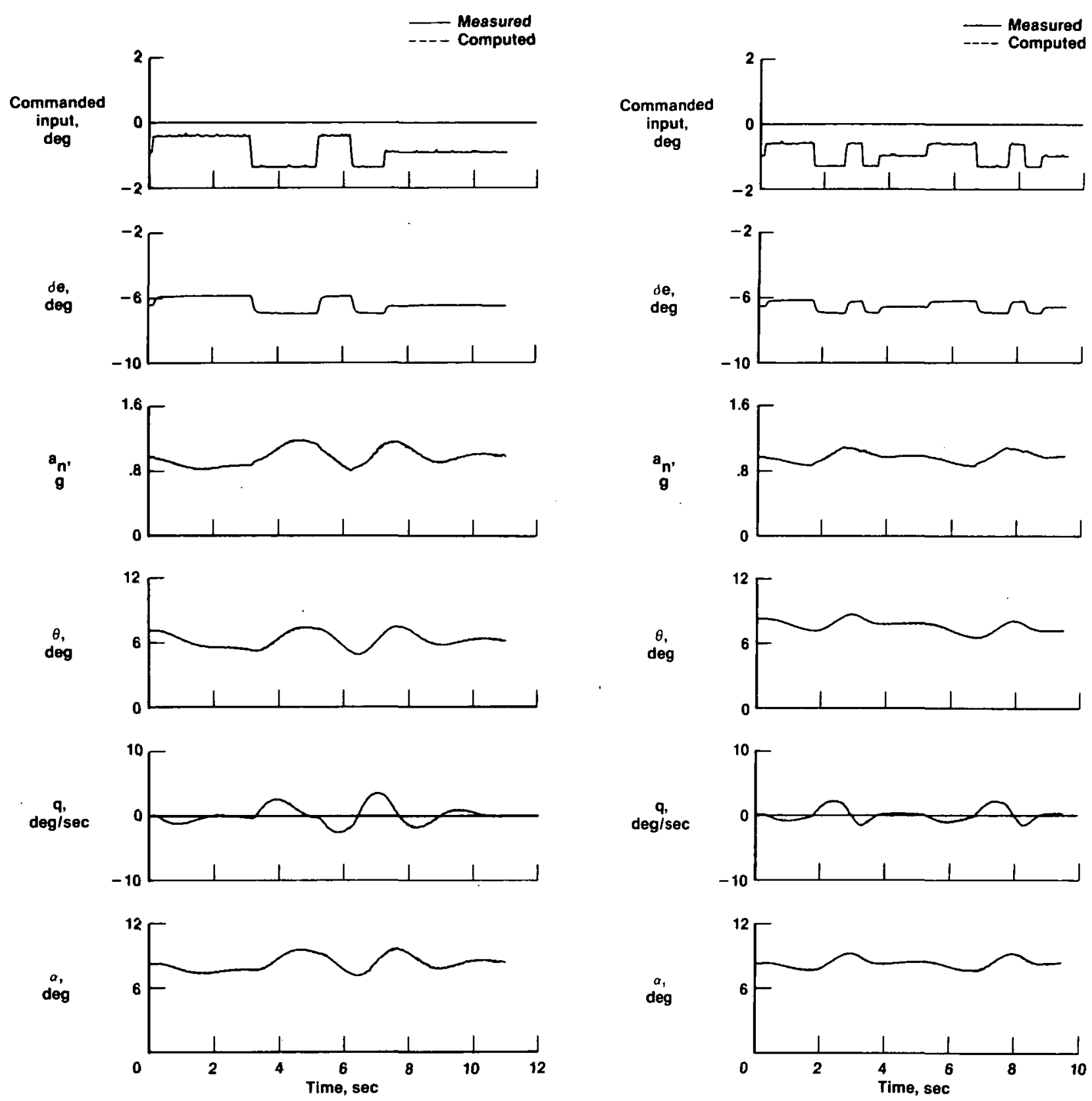

(c) 3211 input (unaugmented mode).

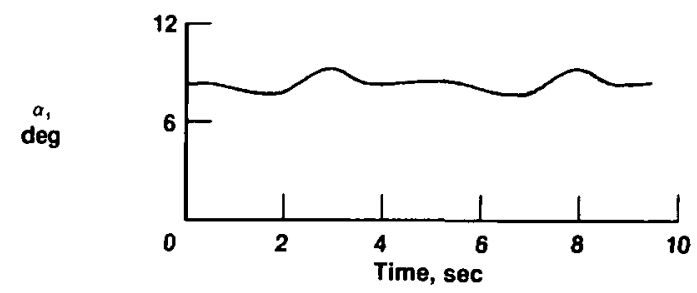

(d) Double 3211 input (unaugmented mode).

Fig. 5 Continued. 

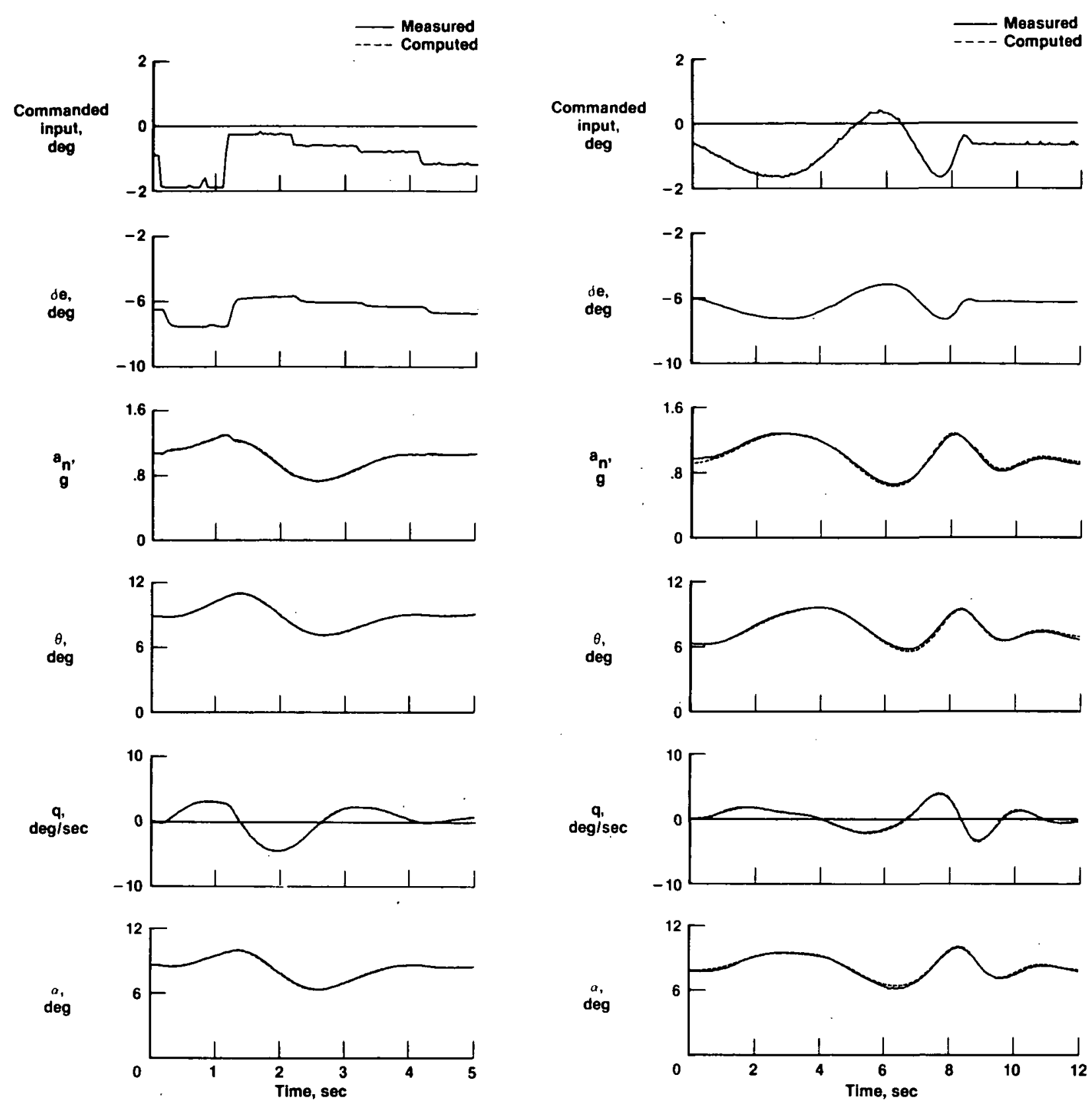

(e) Multistep input (unaugmented mode).

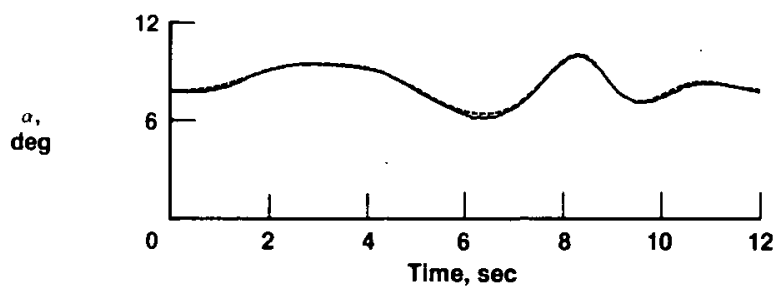

(f) Constant amplitude short sine sweep input (unaugmented mode).

Fig. 5 Continued. 

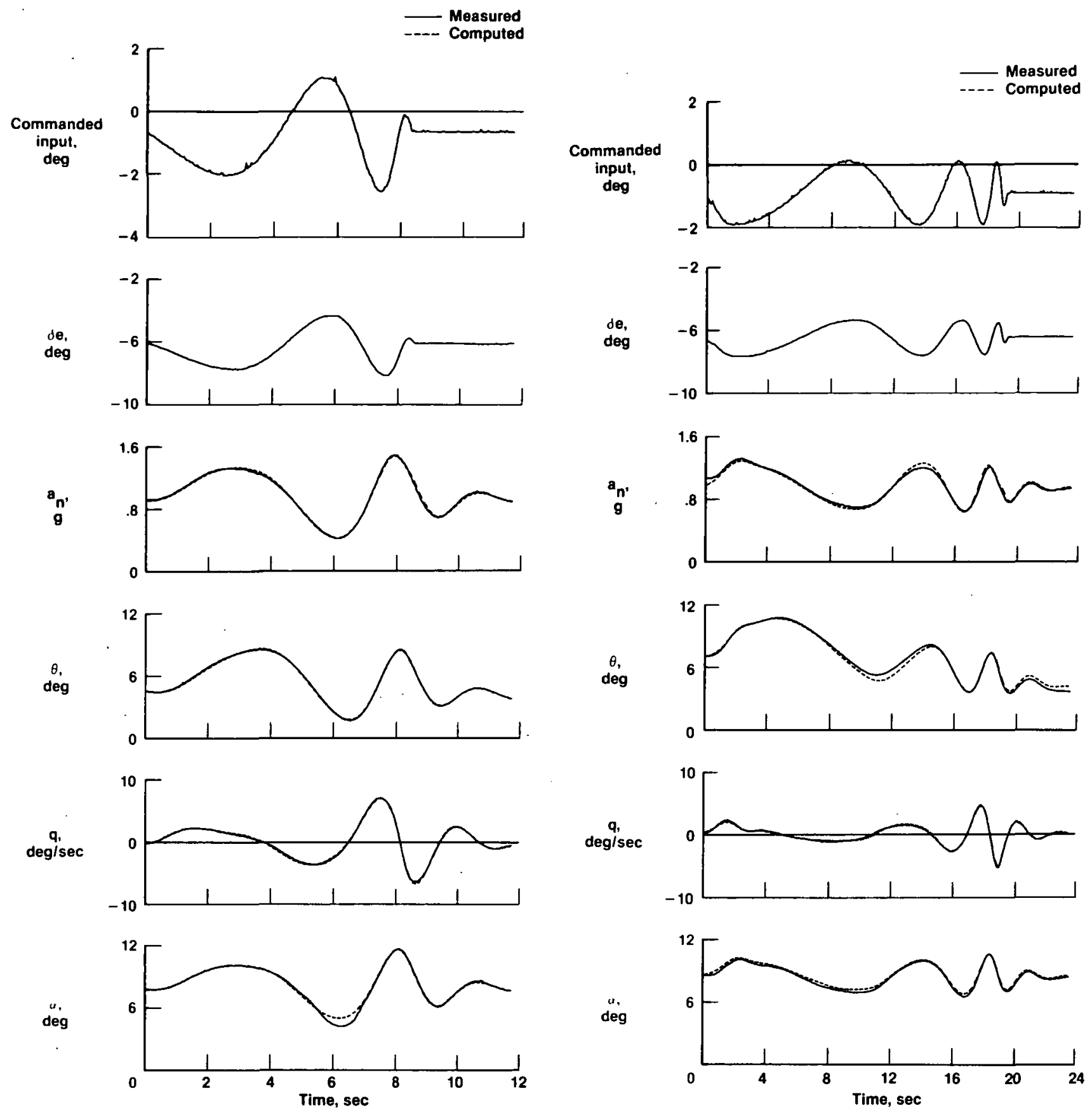

(g) Increasing amplitude short sine sweep input (unaugmented mode).

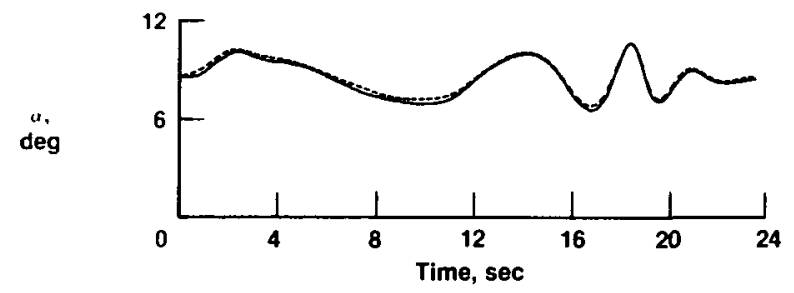

(h) Constant amplitude long sine sweep input (unaugmented mode).

Fig. 5 Continued. 

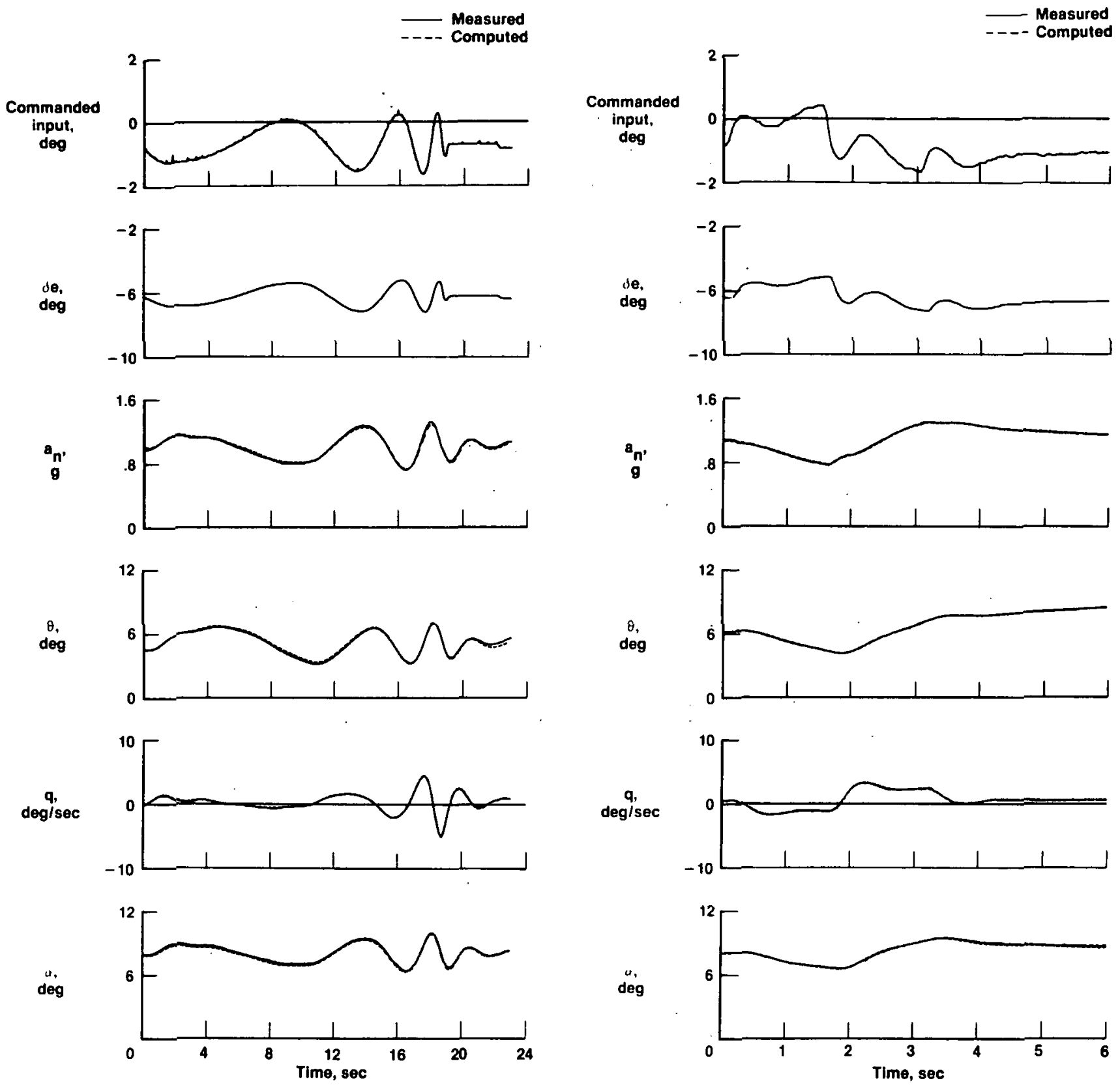

(i) Increasing amplitude long sine sweep input (unaugmented mode).

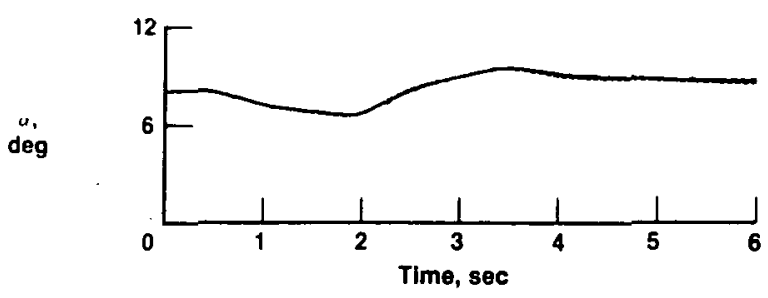

(j) Doublet input (augmented mode).

Fig. 5 Concluded. 


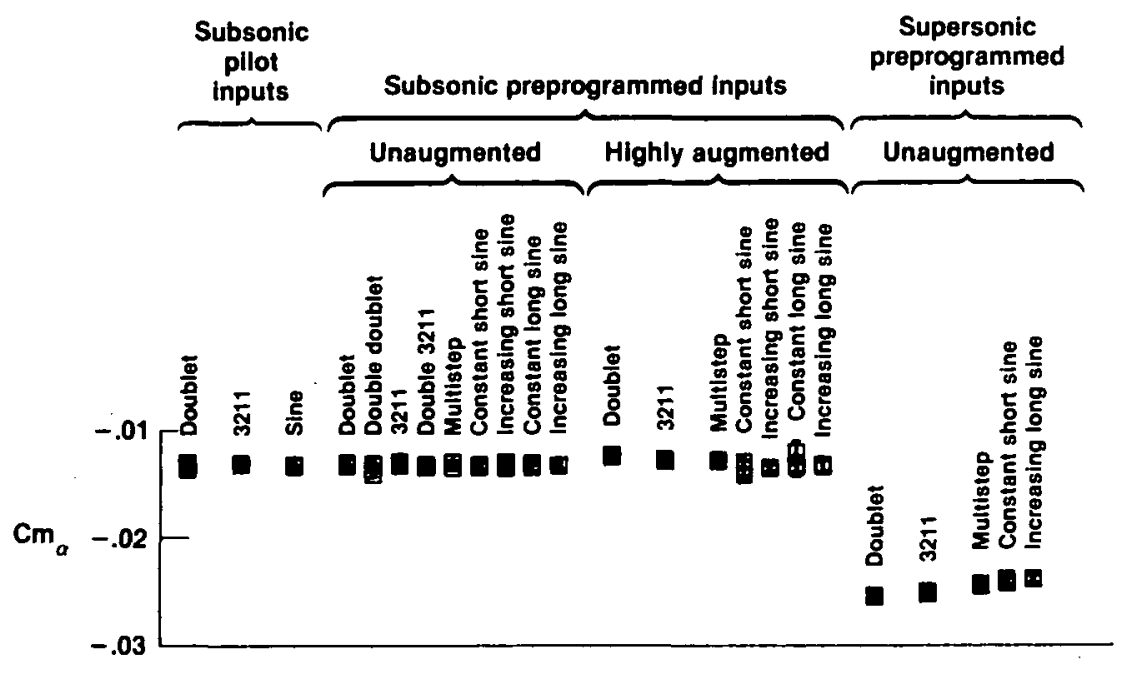

(a) $C m_{\alpha}$.
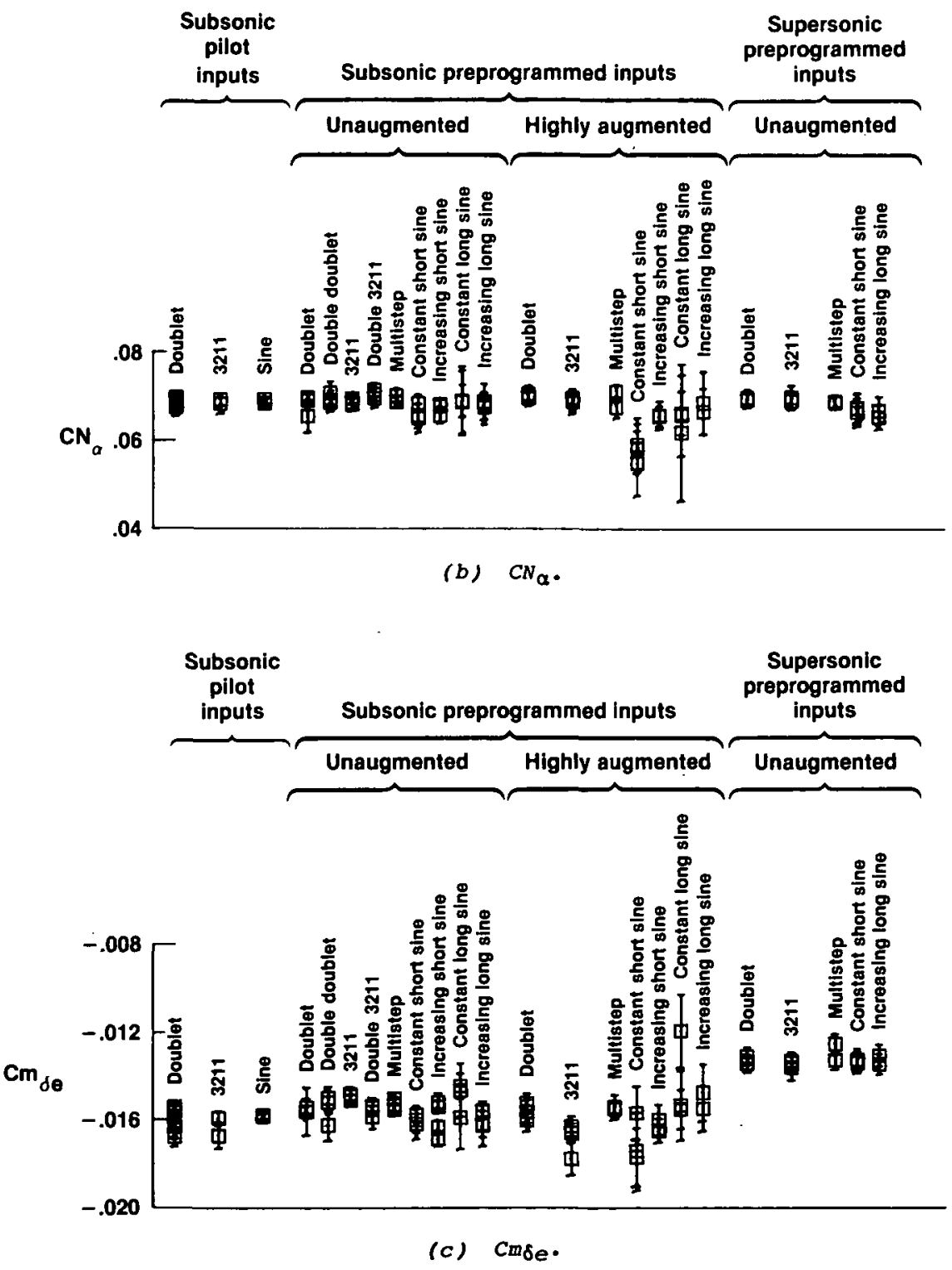

Fig. 6 Summary plots of longitudinal stability and control derivatives. 

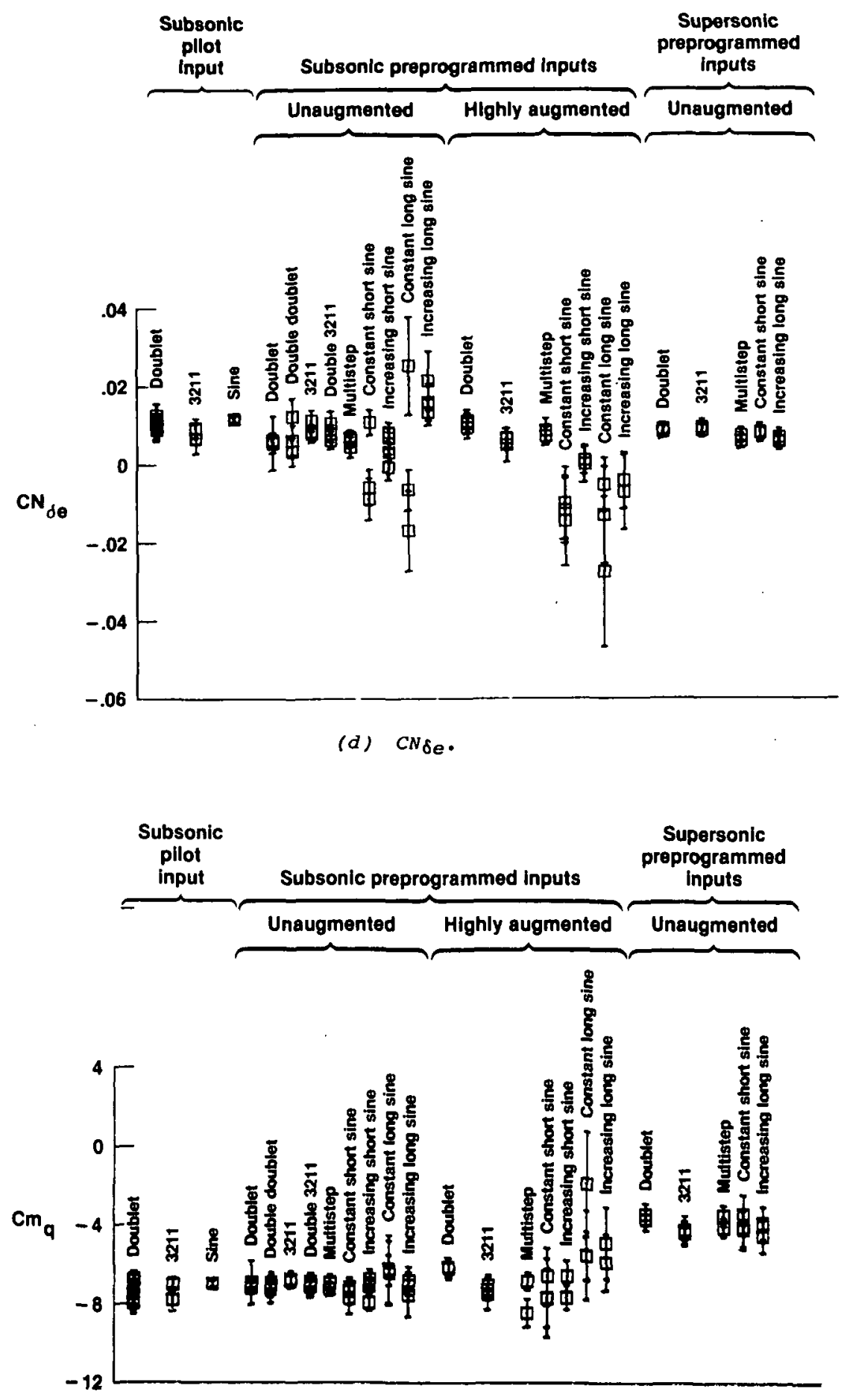

(e) $C m_{q}$.

Fig. 6 Concluded. 


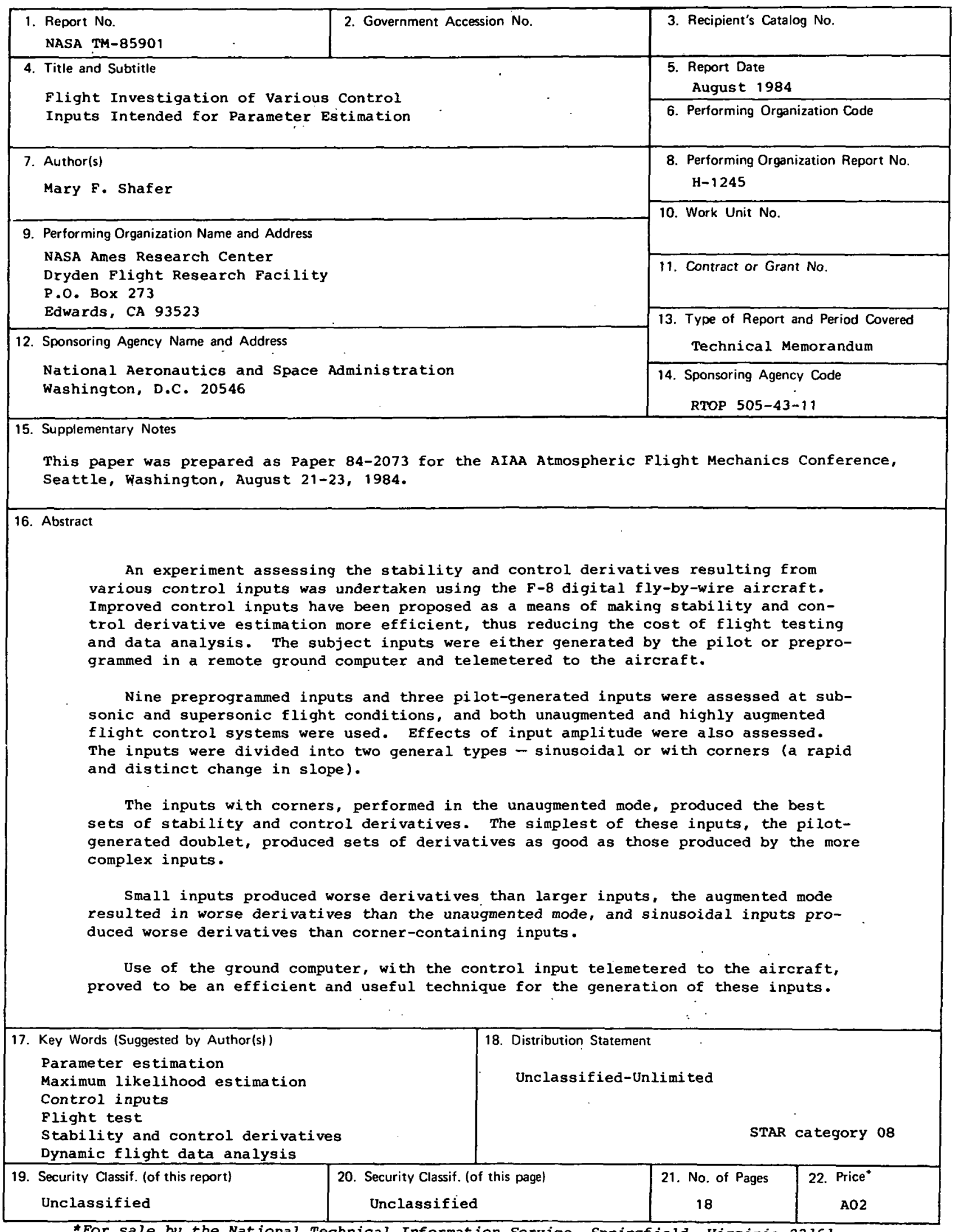

* For sale by the National Technical Information Service, Springfield, Virginia 22161. 